\title{
S-adenosylmethionine, a methyl donor, up regulates tissue inhibitor of metalloproteinase- 2 in colorectal cancer
}

\author{
Z. Hussain ${ }^{1}$, M.I. Khan' ${ }^{2}$, M. Shahid ${ }^{3}$ and F.N. Almajhdi ${ }^{4}$ \\ ${ }^{1}$ Center of Excellence in Biotechnology Research, King Saud University, \\ Riyadh, Saudi Arabia \\ ${ }^{2}$ Department of Inner Medicine I, Medical University of Innsbruck, \\ Innsbruck, Austria \\ ${ }^{3}$ Department of Oral and Maxillofacial Surgery and Diagnostic Science, \\ College of Dentistry, Salman bin Abdul-Aziz University, Alkharj, Saudi Arabia \\ ${ }^{4}$ Department of Botany and Microbiology, College of Science, \\ King Saud University, Riyadh, Saudi Arabia \\ Corresponding author: Z. Hussain \\ E-mail: hussainzahep@gmail.com
}

Genet. Mol. Res. 12 (2): 1106-1118 (2013)

Received July 2, 2012

Accepted September 5, 2012

Published April 10, 2013

DOI http://dx.doi.org/10.4238/2013.April.10.6

\begin{abstract}
DNA methylation is a fundamental epigenetic mechanism in regulating the expression of genes controlling crucial cell functions in cancer development. Gene silencing via $\mathrm{CpG}$ island methylation/ demethylation in the promoter region is one of the mechanisms by which different genes are inactivated/activated in human cancers. Tissue inhibitor of metalloproteinase-2 (TIMP-2) is known to antagonize matrix metalloproteinase (MMP) activity and to suppress tumor growth, angiogenesis, invasion, and metastasis. TIMP-2 expression has been found to be both upregulated and downregulated in various cancers. The inconsistent TIMP-2 expression and unclear epigenetic regulation lead us to investigate its role in colorectal cancer in the presence of a methylating
\end{abstract}


agent. Highly invasive human colorectal cells SW-620 were treated with the methyl donor S-adenosylmethionine (SAM) and its effect was evaluated by cell proliferation, cell cycle, invasion and migration assay. The ability of SAM to down regulate a panel of activated prometastatic, angiogenesis and growth- and cell cycle-regulatory genes was evaluated using end-point and real-time PCR. Treatment of SW-620 with SAM diminished cell proliferation and altered cell cycle kinetic $\mathrm{G}_{2} / \mathrm{M}$ phase cell cycle arrest. An in vitro matrigel invasion assay of SAM-treated cells showed a significant reduction in the invasive potential compared to untreated SW-620 cells. Treatment of SW-620 cells with SAM resulted in activation of TIMP-2 and inhibition of the expression of genes such as MMP (MMP-2, MT1-MMP), urokinase plasminogen activator, and vascular endothelial growth factors. The level of expression of tumor suppressor and apoptotic genes was not significantly higher compared to the untreated control. No changes in the levels of expression of genes (growth and cell cycle regulator), such as TGF- $\beta$, Smad2, Smad4, and p21 were observed. Our data support the hypothesis that TIMP-2, along with other prometastatic genes, is hypomethylated and expressed differently in colorectal cancer. Further in-depth analysis is warranted to confirm the promoter region $\mathrm{CpG}$ methylation pattern of the TIMP-2 gene.

Key words: Colorectal cancer; Epigenetics; DNA hypomethylation; Gene expression; Metastasis

\section{INTRODUCTION}

Colorectal cancer (CRC) is one of the commonest malignant neoplasms with over 1 million new cases and around half a million cancer-related deaths in 2001 (Parkin et al., 2001; McMillan and McArdle, 2007; Jemal et al., 2008). CRC represents a significant global health problem and burden to society especially in the Western world where it represents the second most lethal cancer type (McMillan and McArdle, 2007). The high rate of morbidity and mortality is due to the ability of CRC cells to metastasize to distant organs in the body including the liver (Liotta et al., 1991; Rothbarth and van de Velde, 2005; Bird et al., 2006). Despite recent advances in the treatment options available for various cancers, little headway has been made in either curing or blocking the progression of highly invasive late-stage cancer, resulting in high morbidity and mortality rates (Stetler-Stevenson et al., 1993; Carmeliet and Jain, 2000). The molecular mechanisms responsible for progression to CRC metastasis are largely unknown. An early model postulated that metastasis results from 4 different steps (Liotta et al., 1991; Mollabashy and Scarborough, 2000): i) adhesion of tumor cells to the extracellular matrix (ECM), ii) ability of tumor cells to degrade the ECM and intravasate into surrounding blood vessels, iii) survival against natural defenses, and iv) the formation of new tumors. This is facilitated by the ability of highly advanced cancer cells along with the surrounding stroma to produce several growth factors and proteases that have the ability to inhibit the apoptotic machinery, increase tumor cell proliferation, and cause an increase in new blood vessel (neovascularization) (Mazar et al., 1999; Kerbel, 2008). 
Matrix metalloproteinases (MMPs) are a family of structurally related proteolytic enzymes that facilitate the degradation of ECM and the basement membrane (Liotta et al., 1980; Rao, 2003). High levels of MMP activity have been linked to tumor growth, invasion and angiogenesis (Yamamoto et al., 1996; Gokaslan et al., 1998). Net MMP activity is the result of the balance between the levels of the activated enzymes and tissue inhibitors of metalloproteinases (TIMPs). TIMPs belong to a family of natural inhibitors that control the activity of MMP and are involved in diverse biological processes including cell growth, tumor progression, apoptosis, invasion, metastasis, and angiogenesis (Gomez et al., 1997; Valente et al., 1998). Among the family of TIMPs, TIMP-2 is one of the most frequently investigated members due to its involvement in cancer progression and metastasis (DeClerck et al., 1992; Imren et al., 1996). In contrast to MMPs, which are mostly overexpressed in cancer issue, TIMPs do not show such a consistent expression pattern. TIMP-2 expression was found to be both upregulated and downregulated in different cancers (DeClerck et al., 1992; Valente et al., 1998; Hajitou et al., 2001).

The epigenome, particularly the modifications of cytosines (i.e., methylation) in $\mathrm{CpG}$ dinucleotides, has been gaining great attention as an anticancer target (Razin and Riggs, 1980; Brown and Strathdee, 2002; Esteller, 2005). Abnormal methylation patterns represent a hallmark of cancer (Baylin et al., 2001), and these malignancies are directed by widespread DNA hypomethylation of tumor-promoting genes along with site-specific DNA hypermethylation of tumor suppressor genes (Razin and Riggs, 1980; Baylin et al., 2001; Brown and Strathdee, 2002; Ehrlich, 2002). There is a growing list of genes involved in fundamental cellular pathways and epigenetic regulation (Esteller et al., 2001), and they have the potential to be characterized as epigenetic biomarkers in CRC. This provides an opportunity to explore a panel of genes involved in the metastatic process, especially TIMP-2, which plays a vital role in carcinogenesis. Therefore, we aimed to investigate the role of the methylating agent S-adenosylmethionine (SAM) in the epigenetic regulation of TIMP-2 and its associated genes in CRC.

\section{MATERIAL AND METHODS}

\section{Cell culture and treatment}

Human CRC cell line SW-620 was a kind gift from King Faisal Hospital and Research Center, Riyadh, Saudi Arabia. Cells were grown in alpha minimum medium (alpha-MEM, Gibco-BRL) supplemented with 10\% fetal bovine serum (FBS, Gibco-BRL) and $10 \mu \mathrm{g} / \mathrm{mL}$ gentamicin at $37^{\circ} \mathrm{C}$ in a $5 \% \mathrm{CO}_{2}$ incubator. Treatment with SAM (New England Biolabs) was done by direct addition to regular growth medium under sterile conditions for different days at final concentrations of 75 and $150 \mu \mathrm{M}$. SAM, a methyl donor in DNA methylation reactions, has been previously shown to inhibit replication-independent active demethylation and cause hypermethylation (Shukeir et al., 2006).

\section{Cell proliferation assay}

SW-620 cells were plated in quadruplicate at a density of 60,000 cells/well in $2 \mathrm{~mL}$ of the respective culture medium on 6-well plates. The control and treated cells were grown in regular medium with $3.5 \%$ FBS. The cell growth curve was determined at two different concentrations of SAM ( 75 and $150 \mu \mathrm{M})$. Cell viability was determined by the Trypan blue exclusion 
assay and cell counting was done at different time points starting with day zero without methylating agent using a Coulter Counter (Hertfordshire, UK). Cell culture medium was replenished every day for 6 days along with the indicated concentration of SAM in the treatment group.

\section{Fluorescence-activated cell sorting (FACS) analysis}

The effect of the methylating agent (SAM) on cell cycle distribution of SW-620 was determined by FACS. FACS analysis was done after staining the cells with propidium iodide. The control and SAM-treated (150 $\mu \mathrm{M}$ for 6 days) SW-620 cells were trypsinized and washed with phosphate-buffered saline (PBS), and fixed with chilled $70 \%$ ethanol. The fixed cells were centrifuged and washed with PBS and the cell suspension $\left(10^{6}\right.$ cells $\left./ \mathrm{mL}\right)$ was treated with $1 \mathrm{U}$ DNase-free RNase for $30 \mathrm{~min}$ at $37^{\circ} \mathrm{C}$. Fifty microliters of $1 \mathrm{mg} / \mathrm{mL}$ propidium iodide was added directly to the cell suspension, and flow cytometry analysis was performed on a flow cytometer (FACS Calibur; BD Biosciences). Results were analyzed using the WinMDI software (version 2.8; Scripps Research Institute, La Jolla, CA, USA).

\section{Matrigel invasion assay}

Cells' invasive capacity was determined using a two-compartment Boyden chamber Matrigel invasion assay (Transwell; Costar Corning Corporation, Burlington, MA, USA), as previously described (Shukeir et al., 2006). Polycarbonate filters of a 8- $\mu \mathrm{m}$ pore size were coated with basement membrane Matrigel (50 $\mu \mathrm{g} /$ filter). A total of $5 \times 10^{4}$ cells treated with or without SAM $(150 \mu \mathrm{M})$ in $0.1 \mathrm{~mL}$ medium were added to the upper chamber. This chamber was then placed on top of the lower chamber prefilled with $0.8 \mathrm{~mL}$ serum-free medium supplemented with $25 \mu \mathrm{g} / \mathrm{mL}$ fibronectin (Sigma Aldrich) and then incubated at $37^{\circ} \mathrm{C}$ for $24 \mathrm{~h}$. After incubation, the medium was removed, and polycarbonate filters with invaded cells were fixed and stained. The number of cells invaded was determined under a light microscope, and 10 fields at 400X magnification were randomly selected for analysis.

\section{Cell wounding assay}

Confluent monolayers of SW-620 cells were grown on 6-well plates with $2.5 \%$ FBS. Mechanical wounding was induced in the center of each well with a sterile $200-\mu \mathrm{L}$ pipette tip. Cells were then washed three times to get rid of the detached cells and debris. The cells were then maintained in the presence or absence of SAM $(150 \mu \mathrm{M})$ for $48 \mathrm{~h}$. Wound healing was monitored at different time points with an inverted bright field microscope under a $4 \mathrm{X}$ objective. Only cell-free areas were selected, measured and quantified using the Image Pro-Plus software (Media Cybernetics, Inc., Bethesda, MD, USA), and calculated as percentage wound healing using the equation: \% wound healing $=\left[1-\left(\right.\right.$ wound area at $\mathrm{T}_{\mathrm{xhours}} /$ wound area at $\left.\left.\mathrm{T}_{0}\right)\right]$, where the $\mathrm{T}_{\mathrm{x}}$ is the respective time point and $\mathrm{T}_{0}$ is the time immediately after wounding.

\section{Quantitative real-time RT-PCR}

Total cellular RNA from control and SAM-treated cells was extracted using Trizol (Invitrogen, Life Technologies) according to the manufacturer protocol. Two micrograms of 
total RNA was used for reverse transcription, and cDNA was quantified using end-point PCR, which was confirmed by real-time PCR. The primers used for end-point PCR can be provided upon request while quantitative real-time RT-PCR data are provided in Table S1.

\section{Statistical analysis}

The results are reported as means \pm standard error, and comparisons of the experimental data were analyzed by the two-tailed independent sample Student $t$-test at the 0.05 level of significance.

\section{RESULTS}

\section{Effect of SAM on cell proliferation, cell cycle, invasion, and migration}

In this study, we investigated an approach to inhibit active demethylation in human CRC cells. We measured the effects of two concentrations (75 and $150 \mu \mathrm{M})$ of SAM on cell viability and doubling time. The result presented in Figure 1 shows that both concentrations of SAM significantly decreased cell doubling time. The FACS analysis of cell cycle distribution in control and SAM-treated cells $(150 \mu \mathrm{M}$ for 6 days) revealed a significant increase in the proportion of cells in the $\mathrm{G}_{2}$ phase, with a simultaneous decline in $\mathrm{S}$ phase in the treatment group (Figure 2). Our results showed that SAM treatment of SW-620 significantly decreased cell proliferation by affecting cell cycle $\left(\mathrm{G}_{0} / \mathrm{G}_{1}, \mathrm{~S}\right.$ and $\left.\mathrm{G}_{2} / \mathrm{M}\right)$ distribution.

\section{Proliferation assay}

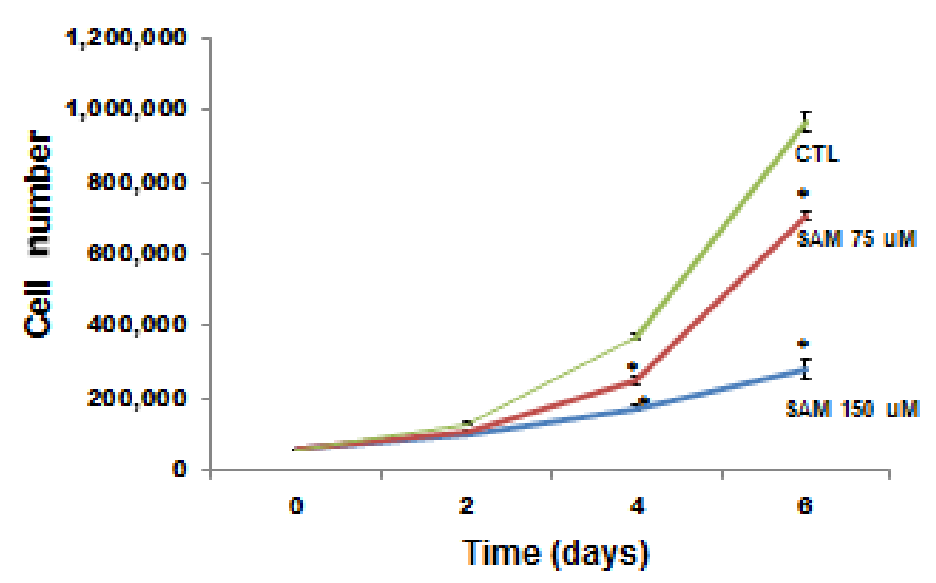

Figure 1. Effect of S-adenosylmethionine (SAM) treatment on proliferation of the invasive cell line SW-620. SW620 cells were seeded at the same density and subjected to two different doses of SAM (75 and $150 \mu \mathrm{M})$ or with vehicle control alone (CTL) on a 6-well plate, and growth curve analysis was carried out to determine the effect of SAM on the proliferation rate. All data are reported as means \pm standard error of quadruplicate wells for each time point. Similar results were obtained for two independent experiments. Significant difference from the control is represented by an asterisk $(* \mathrm{P}<0.05)$. 


\section{Cell cycle}
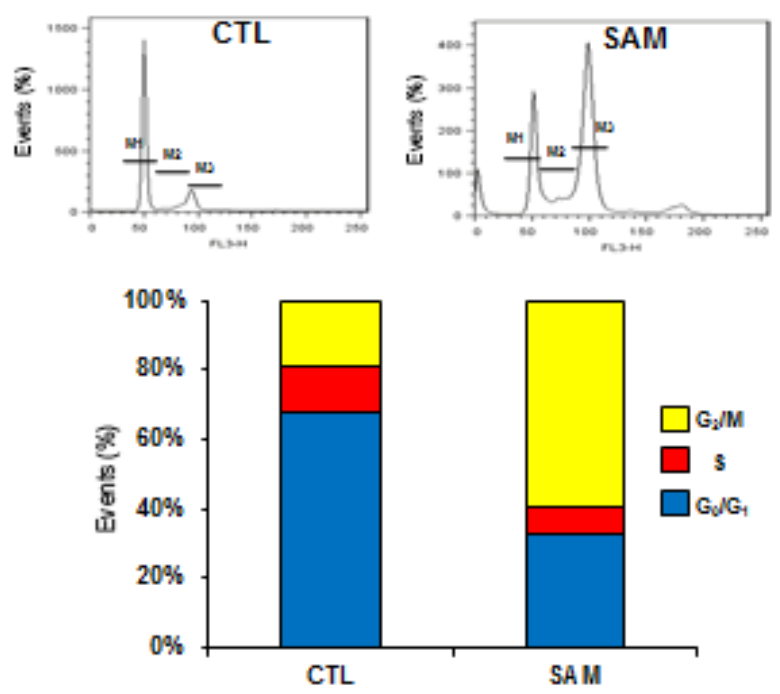

Figure 2. Effect of S-adenosylmethionine (SAM) treatment on cell cycle of the invasive cell line SW-620. Cell cycle distribution analysis was carried out by fluorescence-activated cell sorting for control (CTL) and SAMtreated SW-620 cells (150 $\mu \mathrm{M}$ for 5 days) after staining the cells with propidium iodide. Data were analyzed by WinMDI version 2.8 and plotted and are reported as mean of events (\%) from three independent experiments by $100 \%$ stacked column graph for control and experimental groups.

To determine whether SAM treatment affected the invasive property of the cancer cells, SW-620 cells were treated with $150 \mu \mathrm{M}$ methylating agent. Results from these studies showed a significant $(\mathrm{P}<0.05)$ dose-dependent decrease in tumor cell invasive capacity as determined by the Boyden chamber Matrigel invasion assay (Figure 3). The effect of SAM on cell migration was analyzed, and a significant reduction in wound healing (\%) was observed in SAM-treated $(150 \mu \mathrm{M})$ cells at all times compared with vehicle-treated control cell. These effects were quite pronounced at $24 \mathrm{~h}$ as shown in representative photomicrographs (Figure 4).

\section{Effect of SAM on the genes involved with tumor suppressors, growth factors, apoptosis, and cell cycle}

We tested the hypothesis that SAM diminishes the expression of a series of genes that were previously reported to be involved in cell invasion and metastasis, including MT1-MMP, MMP-2, uPA, and VEGF. SW-620 cells were treated with SAM $(150 \mu \mathrm{M})$ for 6 days. At the end of the experiment, total cellular RNA was isolated and analyzed for the expression of mRNA levels of these genes. These genes were significantly downregulated when treated with methylating agent, as shown in Figure 5. There were nonsignificant changes in the expression of the transforming growth factor- $\beta$ (TGF- $\beta$ ) gene, as well as genes involved in the cell cycle, such as cyclin-dependent kinase inhibitor (p21) and Smad2, Smad4 (Figure 6). The treatment of SW-620 with a methylating agent upregulated the expression of the tumor suppressor genes 


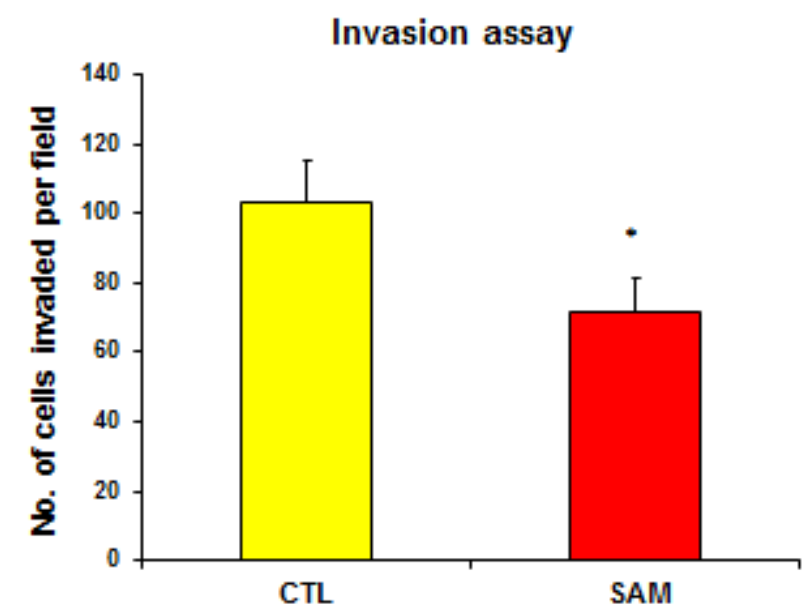

Figure 3. Effect of S-adenosylmethionine (SAM) treatment on invasion of the invasive cell line SW-620. Invasive capacity of SW-620 cells treated with $150 \mu \mathrm{M}$ SAM was determined using a Boyden chamber Matrigel invasion assay. The bar diagram is reported as means \pm standard error as described in Material and Methods. Significant difference from the control (CTL) is represented by an asterisk $(* \mathrm{P}<0.05)$.

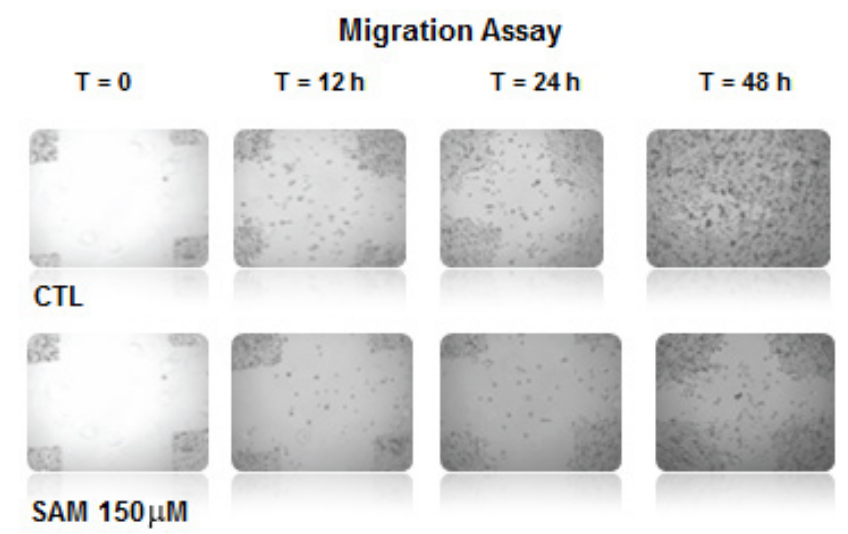

Figure 4. Wound healing migration assay was carried out on SW-620 cells in the absence and presence of 150 $\mu \mathrm{M}$ S-adenosylmethionine (SAM) in the culture medium with $2 \%$ fetal bovine serum. Cells were seeded at the same density and grown as monolayer and wounded as discussed in Material and Methods. Percentage (\%) wound healing was recorded at different time points, and percentage of wound healing with respect to $\mathrm{T}_{\mathrm{o}}$ was calculated using the described equation. Similar results were obtained from two different experiments. All data are reported as means \pm standard error for duplicate values from control (CTL) and experimental groups from two independent experiments.

Wif-1 and RASSF1A, but not to a significant level (Figure 7). As shown in Figure 7, the effect of SAM on Bax, a proapoptotic gene of the Bcl-2 family had a similar mRNA expression level compared to untreated SW-620 cells. Thus, the above results confirmed that SAM was responsible for the downregulation of a battery of prometastatic genes that are normally overexpressed in invasive cell lines. 


\section{Prometastatic and related genes}
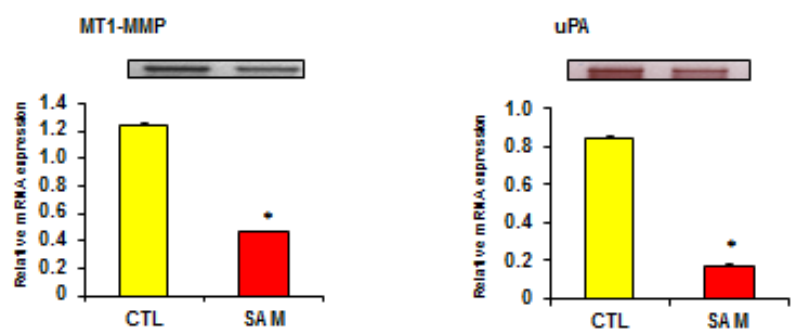

MMP-2
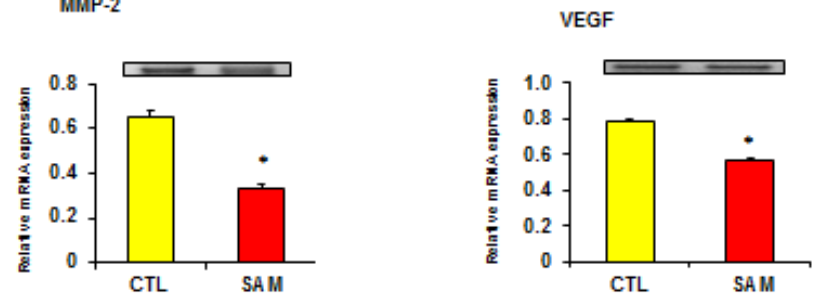

Figure 5. Treatment of SW-620 cells with $150 \mu \mathrm{M}$ S-adenosylmethionine (SAM) deactivates prometastatic and related genes. SW-620 cells were treated with $150 \mu \mathrm{M} \mathrm{SAM}$ for 5 days and at the end of experiment total cellular RNA was isolated with Trizol. RNA from control (CTL) and treatment groups were analyzed for the expression of genes involved in invasion, metastasis and angiogenesis. Changes in mRNA expression of the respective genes were determined by end-point PCR and confirmed by real-time PCR. Significant difference from the control is represented by an asterisk $\left({ }^{*} \mathrm{P}<0.05\right)$.

Cell cycle regulation genes

TGF- $\beta$

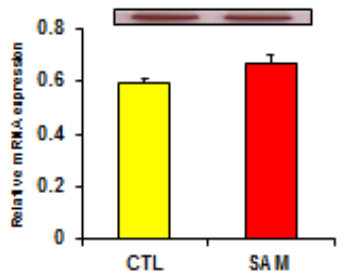

Smad4

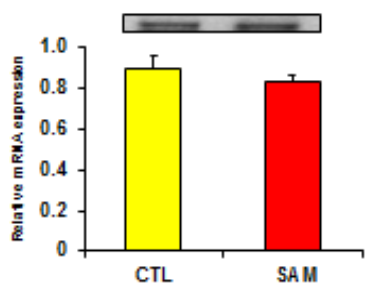

Smad2

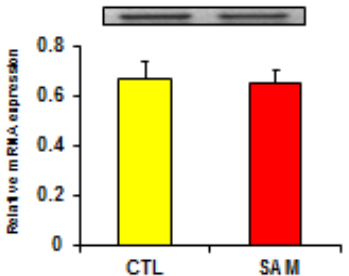

p21

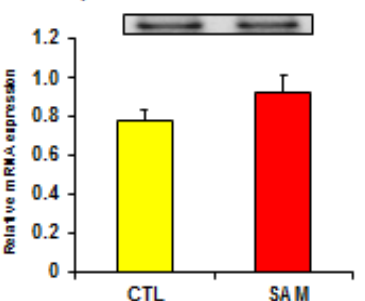

Figure 6. Treatment of SW-620 cells with $150 \mu \mathrm{M}$ S-adenosylmethionine (SAM) deactivates prometastatic and related genes. SW-620 cells were treated with $150 \mu \mathrm{M}$ SAM for 5 days and at the end of experiment total cellular RNA was isolated with Trizol. RNA from control (CTL) and treatment groups were analyzed for the expression of genes involved in cell cycle regulation. Changes in the mRNA expression of the respective genes were determined by end-point PCR and confirmed by real-time PCR. Significant difference from the control is represented by an asterisk $(* \mathrm{P}<0.05)$. 


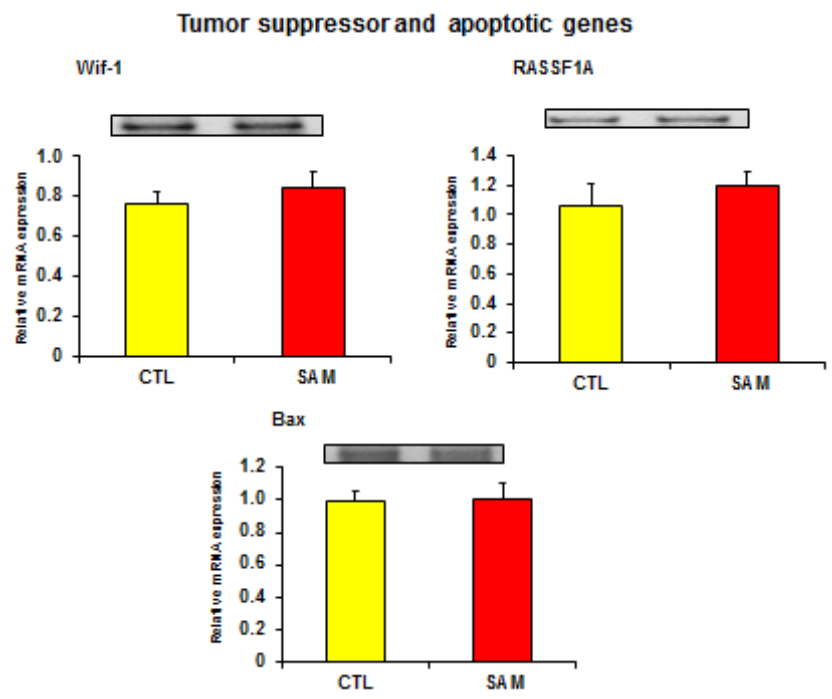

Figure 7. Treatment of SW-620 cells with $150 \mu \mathrm{M}$ S-adenosylmethionine (SAM) activates tumor suppressor and apoptotic genes. Changes in mRNA expression of Wif-1, RASSF1A and Bax1 were determined by end-point PCR and confirmed by real-time PCR. CTL = control.

\section{Effect of SAM on TIMP-2}

Since TIMP-2 plays an important role in regulating tumor angiogenesis and metastasis, we examined mRNA expression of this gene in the presence of SAM. TIMP-2 mRNA expression was upregulated more than 2-fold in the presence of methylating agent (Figure 8). Our results showed that TIMP-2 may be hypomethylated in invasive cancer cells, which need further confirmation by bisulfite sequencing of the promoter region.

\section{TIMP-2}

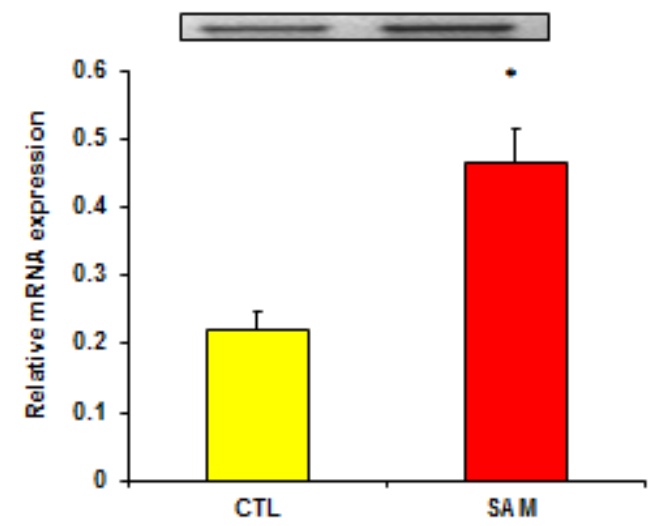

Figure 8. Treatment of SW-620 cells with $150 \mu \mathrm{M}$ S-adenosylmethionine (SAM) activates tissue inhibitor of metalloproteinase-2 (TIMP-2). Changes in mRNA expression of TIMP-2 was determined by end-point PCR and confirmed by real-time PCR. Significant difference from the control $(C T L)$ is represented by an asterisk $(* \mathrm{P}<0.05)$. 


\section{DISCUSSION}

One of the most important prerequisites in developing efficient anti-cancer therapeutics is to block not only primary tumor growth but also tumor metastasis to distant organs (Liotta et al., 1991; Carmeliet and Jain, 2000; Kerbel, 2008). Tumor metastasis is a major cause of concern and is frequently associated with morbidity and mortality. The metastatic cell line SW-620 was used in our study as a model system for identifying genes with a potential role in colorectal metastasis (Futschik et al., 2002). Most of the tumors, including those of CRC, progress using a variety of enzyme systems, where these multipotent enzymes interact with the extracellular matrix and basement membrane. These enzyme systems also play a critical role in tissue remodeling, alterations in cell adhesion and migration, vessel formation and regulation of apoptosis (Liotta et al., 1991; Carmeliet and Jain, 2000; Kerbel, 2008). Numerous cancer studies have postulated that hypomethylation co-regulates a panel of genes involved in metastasis by a common activation mechanism rather than a specific gene (Chiang et al., 1996; Yoon et al., 2001; Detich et al., 2003). The control of these genes through their methylation pattern may prove to be an important strategy in therapeutic efforts. This provides an opportunity to exploit this mechanism by reversal of hypomethylation by hypermethylation therapy (Yoon et al., 2001; Detich et al., 2003; Ateeq et al., 2008). Hypermethylation therapy may provide a unique opportunity for knocking out a whole panel of genes involved in metastasis. This approach may increase the chances for successfully blocking genes, in contrast to therapies that target one protein at a time. Recent data suggest that several genes that are expressed in highly metastatic cells but not in non-metastatic cells are involved in metastasis and are regulated by epigenetic DNA methylation mechanism (Chiang et al., 1996; Detich et al., 2003). In this study, we tested this hypothesis by treating the highly invasive human CRC cells SW-620 with the universal methyl donor SAM to determine if this treatment would result in altered expression of genes involved in colorectal cancer.

The SW-620 treatment with a methylating agent resulted in considerable reduction in cell doubling time as well as the invasive and migratory capacity of these cells. The anti-proliferative and anti-invasive effect of methylating agents can be explained by the hypermethylation of genes involved in the metastatic process. When cells switch from a non-metastatic to a metastatic state, several changes ensue, such as activation of genes (proteases and growth factors) by global hypomethylation (Yoon et al., 2001; Detich et al., 2003; Pakneshan et al., 2004; Ateeq et al., 2008). Selected tumor-promoting genes (proteases and growth factors) were studied in the presence of methylating agent, and we found that SAM deactivated these genes (uPA, MT1-MMP, MMP-2, and VEGF). These results are consistent with the hypothesis that hypermethylation therapy could be a possible approach to late-stage colorectal cancer (Yi et al., 2012). An important concern with using hypermethylation therapy is that it may result in the methylation and silencing of tumor suppressor genes and may thus lead to tumor promotion (Shukeir et al., 2006; Ateeq et al., 2008). One possible consideration that may tone down the potential unwanted effects of hypermethylation therapy is that in several cancer, several genes including tumor suppressor genes are already methylated or partially methylated (Yoon et al., 2001; Detich et al., 2003; Pakneshan et al., 2004; Shukeir et al., 2006; Ateeq et al., 2008). We addressed the question of whether SAM treatment would result in a change in the expression level of these genes. Our results showed statistically nonsignificant changes in mRNA expression of the studied tumor suppressor genes, i.e., Wif-1 and RASSF1A. These findings suggest that inhibition of the hypomethylation machinery could prove to be a novel therapeutic strategy for combating late-stage CRC. 
TGF- $\beta$ is suggested to have biphasic effect on tumor growth; carcinogenesis and early tumor growth are suppressed by TGF- $\beta$, whereas this growth factor apparently accelerates tumor progression in more advanced aggressive tumors (Yin et al., 1999; Kretzschmar, 2000). Therefore, its role in the highly aggressive CRC may be of great importance and needs to be explored in the presence of methylating agent. Our study demonstrated almost no effect of methylating agent on mRNA expression of the TGF- $\beta$ gene, when SAM-treated SW-620 cells were compared to untreated cells. mRNA expression of other genes associated with TGF- $\beta$, such as Smad2, Smad4 and p21, was also not significantly affected by SAM . The diminished role of TGF- $\beta$ and its associated genes may be due to its hypermethylation status in CRC, and hence, there was little or no effect of the methylating agent.

MMPs are mostly overexpressed in cancer tissue; its inhibitor (TIMPs) do not show such a consistent expression pattern. According to different studies, TIMP-2 expression was found to be both upregulated and downregulated in various cancers (DeClerck et al., 1992; Imren et al., 1996; Gomez et al., 1997; Valente et al., 1998; Pulukuri et al., 2007). Recent data identified the downregulation of TIMP-2 expression by epigenetic mechanisms (Suzuki et al., 2002; Ivanova et al., 2004; Galm et al., 2005). Suzuki et al. (2002) observed methylated TIMP-2 in the CRC cell line RKO. However, the methylation of TIMP-2 was not commonly found in primary colorectal tumors. Our study showed a significant increase in gene expression of TIMP-2 when the invasive cell line SW-620 was treated with the methylating agent SAM compared to untreated SW-620. In the current study, the overexpression of TIMP-2 was induced by a methylating agent and thereby limited the invasive characteristics of SW-620. Recently, the overexpression of TIMP-2 has been shown to restrict the invasiveness of various tumor cell types in vitro (DeClerck et al., 1992; Valente et al., 1998). In addition, TIMP-2 overexpression has shown inhibitory effects on tumor growth and angiogenesis in a mouse breast cancer model (Hajitou et al., 2001). However, further in-depth characterization of the CpG methylation status of the TIMP-2 promoter region has to be analyzed to ascertain its prognostic and therapeutic role.

In conclusion, TIMP-2 along with other prometastatic genes are hypomethylated and differentially expressed in colorectal cancer. These genes may play an important role in the inhibition of tumor cell invasion in vitro, and therefore, the $\mathrm{CpG}$ methylation pattern of the promoter region should be determined. To confirm these findings, in-depth in vivo analysis is warranted to prove the epigenetic regulation of these genes.

\section{Conflicts of interest}

The authors report no conflict of interests.

\section{ACKNOWLEDGMENTS}

Research supported by the Deanship of Scientific Research at King Saud University (Project \#RGP-VPP-136).

\section{Supplementary material}

\section{REFERENCES}

Ateeq B, Unterberger A, Szyf M and Rabbani SA (2008). Pharmacological inhibition of DNA methylation induces 
proinvasive and prometastatic genes in vitro and in vivo. Neoplasia 10: 266-278.

Baylin SB, Esteller M, Rountree MR, Bachman KE, et al. (2001). Aberrant patterns of DNA methylation, chromatin formation and gene expression in cancer. Hum. Mol. Genet. 10: 687-692.

Bird NC, Mangnall D and Majeed AW (2006). Biology of colorectal liver metastases: A review. J. Surg. Oncol. 94: 68-80. Brown R and Strathdee G (2002). Epigenomics and epigenetic therapy of cancer. Trends Mol. Med. 8: S43-S48.

Carmeliet P and Jain RK (2000). Angiogenesis in cancer and other diseases. Nature 407: 249-257.

Chiang PK, Gordon RK, Tal J, Zeng GC, et al. (1996). S-Adenosylmethionine and methylation. FASEB J. 10: 471-480.

DeClerck YA, Perez N, Shimada H, Boone TC, et al. (1992). Inhibition of invasion and metastasis in cells transfected with an inhibitor of metalloproteinases. Cancer Res. 52: 701-708.

Detich N, Hamm S, Just G, Knox JD, et al. (2003). The methyl donor S-Adenosylmethionine inhibits active demethylation of DNA: a candidate novel mechanism for the pharmacological effects of S-Adenosylmethionine. J. Biol. Chem. 278: 20812-20820.

Ehrlich M (2002). DNA methylation in cancer: too much, but also too little. Oncogene 21: 5400-5413.

Esteller M (2005). DNA methylation and cancer therapy: new developments and expectations. Curr. Opin. Oncol. 17: 55-60.

Esteller M, Corn PG, Baylin SB and Herman JG (2001). A gene hypermethylation profile of human cancer. Cancer Res. 61: 3225-3229.

Futschik M, Jeffs A, Pattison S, Kasabov N, et al. (2002). Gene expression profiling of metastatic and nonmetastatic colorectal cancer cell lines. Genome Lett. 1: 26-34.

Galm O, Suzuki H, Akiyama Y, Esteller M, et al. (2005). Inactivation of the tissue inhibitor of metalloproteinases-2 gene by promoter hypermethylation in lymphoid malignancies. Oncogene 24: 4799-4805.

Gokaslan ZL, Chintala SK, York JE, Boyapati V, et al. (1998). Expression and role of matrix metalloproteinases MMP-2 and MMP-9 in human spinal column tumors. Clin. Exp. Metastasis 16: 721-728.

Gomez DE, Alonso DF, Yoshiji H and Thorgeirsson UP (1997). Tissue inhibitors of metalloproteinases: structure, regulation and biological functions. Eur. J. Cell Biol. 74: 111-122.

Hajitou A, Sounni NE, Devy L, Grignet-Debrus C, et al. (2001). Down-regulation of vascular endothelial growth factor by tissue inhibitor of metalloproteinase-2: effect on in vivo mammary tumor growth and angiogenesis. Cancer Res. 61: 3450-3457.

Imren S, Kohn DB, Shimada H, Blavier L, et al. (1996). Overexpression of tissue inhibitor of metalloproteinases-2 retroviral-mediated gene transfer in vivo inhibits tumor growth and invasion. Cancer Res. 56: 2891-2895.

Ivanova T, Vinokurova S, Petrenko A, Eshilev E, et al. (2004). Frequent hypermethylation of 5' flanking region of TIMP-2 gene in cervical cancer. Int. J. Cancer 108: 882-886.

Jemal A, Siegel R, Ward E, Hao Y, et al. (2008). Cancer statistics, 2008. CA Cancer J. Clin. 58: 71-96.

Kerbel RS (2008). Tumor angiogenesis. N. Engl. J. Med. 358: 2039-2049.

Kretzschmar M (2000). Transforming growth factor-beta and breast cancer: Transforming growth factor-beta/SMAD signaling defects and cancer. Breast Cancer Res. 2: 107-115.

Liotta LA, Tryggvason K, Garbisa S, Hart I, et al. (1980). Metastatic potential correlates with enzymatic degradation of basement membrane collagen. Nature 284: 67-68.

Liotta LA, Steeg PS and Stetler-Stevenson WG (1991). Cancer metastasis and angiogenesis: an imbalance of positive and negative regulation. Cell 64: 327-336.

Mazar AP, Henkin J and Goldfarb RH (1999). The urokinase plasminogen activator system in cancer: implications for tumor angiogenesis and metastasis. Angiogenesis 3: 15-32.

McMillan DC and McArdle CS (2007). Epidemiology of colorectal liver metastases. Surg. Oncol. 16: 3-5.

Mollabashy A and Scarborough M (2000). The mechanism of metastasis. Orthop. Clin. North Am. 31: 529-535.

Pakneshan P, Szyf M, Farias-Eisner R and Rabbani SA (2004). Reversal of the hypomethylation status of urokinase (uPA) promoter blocks breast cancer growth and metastasis. J. Biol. Chem. 279: 31735-31744.

Parkin DM, Bray F, Ferlay J and Pisani P (2001). Estimating the world cancer burden: Globocan 2000. Int. J. Cancer 94: 153-156.

Pulukuri SM, Patibandla S, Patel J, Estes N, et al. (2007). Epigenetic inactivation of the tissue inhibitor of metalloproteinase-2 (TIMP-2) gene in human prostate tumors. Oncogene 26: 5229-5237.

Rao JS (2003). Molecular mechanisms of glioma invasiveness: the role of proteases. Nat. Rev. Cancer 3: 489-501.

Razin A and Riggs AD (1980). DNA methylation and gene function. Science 210: 604-610.

Rothbarth J and van de Velde CJ (2005). Treatment of liver metastases of colorectal cancer. Ann. Oncol. 16: 144-149.

Shukeir N, Pakneshan P, Chen G, Szyf M, et al. (2006). Alteration of the methylation status of tumor-promoting genes decreases prostate cancer cell invasiveness and tumorigenesis in vitro and in vivo. Cancer Res. 66: 9202-9210.

Stetler-Stevenson WG, Aznavoorian S and Liotta LA (1993). Tumor cell interactions with the extracellular matrix during invasion and metastasis. Annu. Rev. Cell Biol. 9: 541-573. 
Suzuki H, Gabrielson E, Chen W, Anbazhagan R, et al. (2002). A genomic screen for genes upregulated by demethylation and histone deacetylase inhibition in human colorectal cancer. Nat. Genet. 31: 141-149.

Valente P, Fassina G, Melchiori A, Masiello L, et al. (1998). TIMP-2 over-expression reduces invasion and angiogenesis and protects B16F10 melanoma cells from apoptosis. Int. J. Cancer 75: 246-253.

Yamamoto M, Mohanam S, Sawaya R, Fuller GN, et al. (1996). Differential expression of membrane-type matrix metalloproteinase and its correlation with gelatinase A activation in human malignant brain tumors in vivo and in vitro. Cancer Res. 56: 384-392.

Yi JM, Dhir M, Guzzetta AA, Iacobuzio-Donahue CA, et al. (2012). DNA methylation biomarker candidates for early detection of colon cancer. Tumour. Biol. 33: 363-372.

Yin JJ, Selander K, Chirgwin JM, Dallas M, et al. (1999). TGF-beta signaling blockade inhibits PTHrP secretion by breast cancer cells and bone metastases development. J. Clin. Invest. 103: 197-206.

Yoon SO, Kim MM and Chung AS (2001). Inhibitory effect of selenite on invasion of HT1080 tumor cells. J. Biol. Chem. 276: 20085-20092. 